d) To co-ordinate the use of isolated instruments existing in various institutions.

e) To improve astronomy education through: education of high school teachers, development of high school curricula, conferences, seminars, radio and TV programs etc.

Finally, the proposed relocation of one of the Vatican Observatory telescopes in Peru will be a happy opportunity to form a Peruvian astronomical center.

\title{
THE SUMMER SCHOOLS OF THE VATICAN OBSERVATORY
}

\author{
Martin McCarthy \\ Vatican Observatory, Vatican City State
}

Starting on the 10th of June, 1986, the staid and quiet halls and courtyard and corridors, including the giant circular staircase designed by Bernini for the little donkeys that carried Popes to their quarters in the summer palace, echoed to the swift patter of lightfooted students and the buzz of their conversations as 17 young men and 8 young women met at the first Vatican Observatory Summer School in Astronomy and Astrophysics at Castel Gandolfo.

These 25 scholars had been chosen from a list of 135 candidates at university and graduate school campuses all over this planet. More than 30 candidates were rejected for "excellence"; they were judged to be too far advanced for admission to the classes on Galaxies and Dark Matter and to those on Spectral Classification and on Instrumentation for Photometry and Image Processing. The classes were aimed at students just beginning or planning immediate entrance into graduate level classes; the School was not planned for "new Ph.D.s," for "post-docs," or for those already well into thesis work. Criteria for admission included academic grades, recommendations of university professors, plus personal statements from the candidates on reasons why they felt they wanted to attend the sessions of the school. Applications were studied by faculty and staff members and results were announced in January, giving students some four months to arrange their travel and home commitments so they could be free to respond to the school bells on June 10 .

It was decided early on to assign a majority of places to young scholars from the developing nations on our planet. Fourteen came from homes and schools there, while eleven voyaged to Castel Gandolfo from industrialized nations. Neither race nor creed nor sex were discriminants.

Three students each came from India, Argentina, Italy and the United States; and two each from Greece, New Zealand, Brazil, and Argentina. China, Austria, Korea, Bangladesh, Yugoslavia, Holland, Belgium, Poland, and Nigeria had one representative each. English was the required language for admission to the school. 
Conversations through the days and nights were conducted in many diverse tongues and on the blackboard used to welcome the members of the Vatican Press Corps, who had asked to meet the students, greetings were inscribed in 23 languages.

Two features of the school merit mention here. The generosity and concern of the Holy Father made our orientation towards the Third World both possible and practical. For all students, free tuition and free registration were offered and there were no fees assigned for laboratory use, for the libraries, for stationery supplies, or for computer programming. In addition, coffee and croissants (the latter quite a rarity in the papal palace) were offered each schoolday and a "free lunch," Italian style, was served to all students and faculty on the lovely palace terrace above the volcanic crater lake called Albano and looking towards the $900-\mathrm{m}$ (3000-foot) peak of Monte Cavo. For the students from the developing nations (14 of 25), the Holy See paid for air transport plus 70 per cent of board and room. Travel and lodging could not be offered to students from the industrialized countries, but the Local Organizing Committee headed by the director of the Vatican Observatory, Dr. G.V. Coyne S.J., was able to find and arrange for inexpensive housing facilities for these also.

The second feature of this Summer School in 1986 was noted by the Holy Father in the address he gave to the students on 30 June, the eve of his departure for Colombia in Latin America; he declared that the school was possible in large part because of the significant gift of the professors' lectures, which were offered without salary. The Vatican covered travel expenses for the professors and their spouses and hosted them during the period of the school.

The professor who lectured most frequently in the school was Dr. Vera Rubin of the Carnegie Institution of Washington, whose lectures on Galaxies and Dark Matter in the Universe ran through the entire month. The Dean of the School, Dr. Martin McCarthy S.J. of the Vatican Observatory Staff, and Prof. David Latham of the Harvard-Smithsonian Center for Astrophysics divided the lecture set that ran parallel to Dr. Rubin's. McCarthy lectured on Spectral Classification of Stars with application to problems of galactic structure; Latham described new observational techniques as used in current problems in astrophysics. Supplementary lectures were given by Dr. Coyne and by Dr. V. Piirola, visiting research professor from Helsinki University; they described for the students some of their exciting research in polarimetry of the interstellar medium; Dr. C. Corbally of the Vatican Observatory Staff gave two lectures on Stellar Evolution. These main lectures were given each morning in the classroom, which had once contained the large grating and prism spectrographs used by the Vatican team of Gatterer, Junkes, and Salpeter in making the world-famed Vatican Spectral Atlases found in physics labs and in observatories the world over. The first class ran from 9:00 am to 10:30 am, the second from 11:00 am to $12: 30 \mathrm{pm}$. Labs and special lectures by visiting scientists plus laboratory research and library consultation for assigned projects occupied the post-prandial period, while the late afternoon and early evenings were kept free for recreational activities (swimming, tennis, jogging, cycling, etc.).

Evaluation is a long process and continued throughout the ensuing months. 
The students seemed to have profited by their working and studying and playing together; a common complaint was that the school term was too short. All of us in planning the school realized that this brevity was necessary, since the summertime for students and professors from northern and southern latitudes could not allow more extensive time away from home institutions. Further, here at the Vatican the coming of the Holy Father for his annual and much needed vacation meant that the scholarly (and sometimes noisy) activities of young scholars had to be completed before the palace became the center for the organizational, diplomatic, and spiritualcultural events that always mark the papal visits to Castel Gandolfo.

On 6 June 1988, bells rang again at Castel Gandolfo for the second session of the Vatican Observatory's Summer School. Yes, it was the same and yes, there were differences between the two sessions. Same was the number of students, same was the emphasis on the international composition of the student body, and similarly the solid encouragement for young scholars from developing nations. The appendix gives the enrollment statistics for the 1986 and 1988 Summer Schools. The differences? This year there were two Deans: Prof. Charles Lada of the Steward Observatory of the University of Arizona and Dr. Christopher Corbally S.J. of the Vatican Observatory Research Group, also located in Tucson, Arizona. The co-deans soon enlisted the professional aid of Dr. Jean Keppel of the Kitt Peak National Observatory and Prof. Frank Shu of the University of California in Berkeley to be their colleagues in the principal teaching roles at the school. These four, with consultation from Dr. George Coyne S.J., who was also residing in Tucson in connection with his regular teaching role at the University of Arizona, were able together to decide on the composition of the student body and to initiate communication with the young scholars selected for Summer School '88. The topics for the lectures in 1988 differed from those offered two years previously. Only Galaxies remained a common feature for the two schools. Star Formation and the Interstellar Medium replaced Spectral Classification and Stellar Photometry as lecture topics.

Another difference concerned the activities outside the two formal morning lectures from 9:00 am to $12: 30 \mathrm{pm}$. In 1986, activities had included the laboratory, library, and computer exercises concerned chiefly with practical progress in classifying and analyzing galaxies and their stellar composition and in spectral classification from the Anglo-Australian 1.2-m (48") Schmidt photographs. In contrast, the scholars of 1988 were invited during the opening days of the school to report formally on the research work that they had been engaged in at their home institutions; this consisted of 10-minute reports (with warnings at 8 minutes) made to their fellow students under the moderation of Dean Corbally and Dean Lada. Then, at the close of the 1988 school days, the students, now in pairs (with no two scholars from the same country allowed to collaborate), presented 20 -minute talks before the class, the faculty, and observatory staff members. Topics had been assigned by the professors, and the results were presented orally by each student in turn with slides and transparencies; each talk was followed by questions from the audience. The present writer, as former Dean, was very impressed with the high level of student reporting: they seemed to have mastered the art of literature searching and demonstrated clear 
writing skills and presentation techniques plus a quite surprising maturity in their evaluation of scientific projects. Of course, many of the faults that beset scientific presentations even at a much higher level of expertise could be (and were) noted (and pointed out to the speakers). Some showed a tendency to speak too rapidly, some too softly; some transparencies appeared too crowded and data-dense. Castel Gandolfo seemed to be a very good place to start and we hope the students will look back on these first steps in public presentation of science results with joyous recollections they move on to higher things in the future: reports on their own research.

Another difference noted between the 1986 and 1988 versions of the Vatican Observatory's Summer Schools was an increased mental and verbal familiarity that the students of 1988 showed at the end of their courses with the fundamental notions of stellar evolution and star formation. The interactions of observations and theory had been well presented to them and they seemed ready for this and were not "snowed" or "overawed" by the formulations of the transfer equations or by the laws of radiation and absorption.

The length of the school, 30 calendar days, was for the Director, Deans, and Staff a non-variable that cannot be altered for the reasons stated above. Hence also formal examinations, written or oral, and grading of students were not part of the plan. Opportunities for student-teacher interaction were many and easy to arrange; other exercises in visual observations were offered and accepted, with Dr. E. Carreira S.J. using the $40-\mathrm{cm}$ visual Zeiss refractor on the palace roof. Alas, the telescopes, including a $60-\mathrm{cm}$ reflector, and a $40-\mathrm{cm}$ four-lens refractor, the Vatican 63/93/250$\mathrm{cm}$ Schmidt, and the ancient Carte Du Ciel camera, located in the papal gardens of the Villa Barberini, have long been "light-struck" by the growing industrial complex on the Roman plain some $450-\mathrm{m}$ ( 1500 feet) below the observatory and are no longer usable for modern research problems. We note here that the courses offered were lecture-based and not observing-based; in large part this method was used because of the brevity of our school season and in part because of the deterioration of the Roman skies.

One worthwhile feature common to both the 1986 and 1988 summer schools that bids fair to be continued and extended in future sessions is the scheduling of historical talks and visits. Invited talks were given by visitors and staff members in both the 1986 and 1988 sessions; topics ranged from archeoastronomy to relativistic astrophysics; a strong emphasis was accorded to the life story and problems of Galileo Galilei, as seemed most fitting for a school in Italy and in the palace built by Pope Urban VIII, who so greatly admired Galileo and later imposed so harsh a penalty on him. Together, faculty, staff, and students visited sites of historical scientific interest in Florence, Arcetri, Padua, and Venice on "break-day" during one weekend of each session. It seemed to the Faculty of the School to be most fitting to offer some introduction to what we hoped might be an early and continuing historical interest among our young scholars. In 1986, as guests of the Vatican Observatory, students of the School went together with the faculty by buses to Florence and Arcetri, where we saw at close range the "roba di Galileo"; in 1988 two 
other "Galilean" cities - Padua and Venice - were visited, this time via overnight train-couchettes plus a one-day stay at an excellent and central Casa del Pellegrino in Padua. At the Galilean sites in Arcetri, Florence, and Padua, we were guided and helped by our astronomical colleagues from the observatories of Padova-Asiago and Arcetri.

There were also several visits to historical scientific sites in Rome. Through these we hope that our young scholars can begin early to reflect on the beginnings and early developments in science. Perhaps in the future one could think of "break day" visits to the north of Italy and someday even to the lands of Pythagoras and his fellows in Calabria and Sicily. No, indeed we cannot even imagine transporting the whole Summer School troop for a short and glorious visit to Greece, even though all of the young scholars from Greece both in 1986 and 1988 have been most enthusiastic in suggesting this.

Now we will see how our young scholars mature and grow and develop in their chosen scientific work. We shall look to see how they in their turn will help younger brothers and sisters along the rugged pathways that lead to the stars. We hope that they will remember the last words that the Holy Father spoke to the students in 1986, the same ones that are written in marble on the walls of the Observatory at Castel Gandolfo: Deum Creatorem Venite Adoremus: Come let us adore God our Creator and Lord.

\section{Appendix}

Nations sending scholars to Vatican Observatory Summer Schools in 1986 and 1988:

Argentina, Austria, Bangladesh, Belgium, Brazil, Canada, China, Finland, Great Britain, Greece, Holland, Hungary, India, Indonesia, Italy, Korea, Mexico, New Zealand, Nigeria, Poland, Sri Lanka, Turkey, United States, Venezuela, Yugoslavia.

Total: 25 nations, 14 developing nations, 11 industrialized nations.

Scholars at Vatican Observatory Summer Schools: 1986 and 1988

\begin{tabular}{lccc} 
Scholars & In 1986 & In 1988 & Totals \\
\hline $\begin{array}{l}\text { From } \\
\begin{array}{l}\text { Developing } \\
\text { Nations }\end{array}\end{array}$ & 25 & 25 & 50 \\
$\begin{array}{l}\text { From } \\
\text { Industrialized }\end{array}$ & 14 & 16 & 30 \\
Nations & & 9 & 20 \\
Women & 8 & 8 & 16 \\
Men & 17 & 17 & 34 \\
\hline
\end{tabular}




\title{
Discussion
}

\section{D.G. Wentzel: What happens to the Schmidt and other telscopes?}

M. McCarthy: The research telescopes at Castel Gandolfo (see Annual Report of Vatican Observatory 1963, D.J.K. O'Connell) are "lightstruck" by the growth of suburban Rome with its large industrial parks in the Roman plain. The Vatican Observatory Research Group moved to Tucson, Arizona, U.S.A., five years ago, where the new 1.8-m telescope will be erected (squirrels permitting) on Mt. Graham, Arizona. Dr. George Coyne, Director of the Observatory is directing the disposal and transfer of available telescopes. At present he is working with colleagues from Peru concerning the moving of the Zeiss doublet south of the equator.

Ed. note: There is uncertainty whether Mt. Graham will be allowed to be used as a telescope site for environmental reasons, including whether a certain type of rare squirrel there will be affected.

\section{SURPLUS TEXTS AND JOURNALS FOR ABROAD - A CANADIAN PROGRAM}

\author{
Alan H. Batten \\ Dominion Astrophysical Observatory, Victoria, British Columbia, Canada \\ Dieter W. Brückner \\ Department of Astronomy, University of Toronto, Toronto, Ontario, Canada
}

We wish to report briefly on a program for sending donated astronomical books and journals to overseas institutions that have not been able to acquire them in other ways. The program is being sponsored for a trial year by the Canadian Astronomical Society (CASCA), in co-operation with the Canadian Organization for Development through Education (CODE), which has generously offered to pay for the shipments to some countries, mostly in Africa and the Caribbean.

We have been soliciting donations from among the membership of CASCA for materials surplus to their needs, and have offered these through the Newsletter of IAU Commission 46, the IAU Bulletin, and Colloquium 105. Our efforts have been successful, and at the time of writing, about half the journal runs and texts, and almost all of the monographs available at the time of the Colloquium have found recipients ${ }^{1}$. We are gratified by this result, as we had been unsure of the usefulness of what we were doing. Discussions at the Colloquium have given us additional

\footnotetext{
${ }^{1}$ in Bulgaria, Poland, India (2), Egypt, and Nigeria.
} 